\title{
Holismus und Perspektivismus in Hegels Auffassung der Willensfreiheit
}

\author{
Holism and perspectivism in Hegel's \\ understanding of the Free Will \\ JAKUB KLOC-KONKOŁOWICZ \\ Instytut Filozofii Uniwersytetu Warszawskiego (Varsovia)
}

Recibido: 10-03-2014 Aceptado definitivamente: 01-04-2014

\begin{abstract}
ZUSAMMENFASSUNG
Hegelsche Philosophie wird oft als eine metaphysische Konstruktion angesehen. Demgegenüber wird in dem Beitrag eine Position eingenommen, laut der Hegel, innerhalb seines holistischen philosophischen Rahmens, eine perspektivistische Vorgehensweise verwendet, um immer neue, immer konkretere, weil begrifflich immer mehr vermittelte, Einsichten zu erreichen. Die perspektivistische Methode Hegels wird anhand des Phänomens des freien Willens gezeigt. Hegel glaubt, dass es sich nicht auf einen Schlag erklären lässt, ob und in welchem Sinne der Wille frei ist. Der Beweis, der diese Versicherung begründen soll, wird erst auf dem Weg der vielschichtigen philosophischen Deduktion geliefert. Einer neuen Auffassung des Trägers der Willensfreiheit entspricht dabei immer ein neues Konzept des Gegenstandbereiches. So impliziert etwa das moralische Konzept des Willens eine völlig neue begriffliche Konstellation, mit welcher der Wille und seine Welt aufgefasst wird, als das rechtliche Konzept des Willens. Im letzten Teil des Beitrags wird analysiert, welche Schlüsse aus Hegelscher Rekonstruktion des freien Willens für heutige Debatten zu ziehen wären. Demgemäß sollte ein hegelianisch gesinnter Denker nicht das von dem Naturalisten entworfene Bild des empirisch determinierten Willens, sondern dessen absoluten Anspruch auf Erklärung desavouieren. Es wird klar, dass empiristische Vorgehensweise im Fall der Willensfreiheit einen gewaltigen Teil unserer sozialen Erfahrung und unseres Diskurses gar nicht erklären kann. Mann sollte die theoretische Ebene vertiefen (sie um moralische, rechtliche, so
\end{abstract}

Suplemento 19 (2014)

(C) Contrastes. Revista internacional de filosofía, pp. 75-94. ISSN: 1136-9922

Departamento de filosofía, Facultad de Filosofía y Letras, Universidad de Málaga Campus de Teatinos, E-29071 Málaga (España) 
ziale Perspektiven bereichern), auf der die Polemik mit den naturalistischen Gegnern der Willensfreiheit aufzunehmen ist.

SCHLÜSSELBEGRIFFE:

FREIHEIT, WILLE, PERSPEKTIVE, HOLISMUS, MORALITÄT

\begin{abstract}
Hegel's Philosophy is often seen as a metaphysical construction. Contrary to that, the author of the paper tries to show that Hegel uses - in the context of his holistic philosophy - a perspectivist strategy which allows him to present newer and more and more detailed insights, which are conceptually richer. This perspectivist strategy of Hegel is demonstrated using the example of the problem of the freedom of will. According to Hegel there is no possibility to show at one blow that, and in what sense, the will is free. The argument that vindicates this assumption can only be given in the context of multi-layered philosophical deduction. A new concept of the subject of the free will always implicates a new concept of the objective sphere. For example, the moral understanding of the will implies a wholly new configuration of concepts to grasp the will and its world than the legal understanding of it. At the end of the paper an analysis is given of what kind of conclusions may be drawn from Hegel's reconstruction of the free will that could bring new insights in the actual debates on the free will. According to this analysis a hegelian thinker should not disavow the naturalist's picture of the empirically determined will, but should instead disavow the claim of this picture to have an absolute explanatory status. It is suggested that the empirical strategy of the denial of the free will is not able to explain a huge range of our social experience and our social discourse. The theoretical level on which the discussion with a naturalistic opponent to the freedom of will is being undertaken should be deepen, i.e. enriched with moral, legal and social perspectives.
\end{abstract}

\title{
KEYWORDS \\ FREEDOM, WILL, PERSPECTIVE, HOLISM, MORALITY
}

Über Perspektivismus in der Philosophie von Georg Hegel zu sprechen, ist ein kontroverses Unterfangen. Denn für viele Hegel-Interpreter, besonders für diejenigen, die sich kritisch mit seinem Denken auseinandersetzen, gilt als sicher, dass das Hegelsche Projekt der Philosophie in einem absolutistisch-metaphysischen Rahmen eingeschlossen ist und dass Hegel gerade für sich selbst den absoluten Standpunkt - den Standpunkt, der selber nicht mehr perspektivistisch ist, sondern sich über jede partielle Perspektive erhebt - beansprucht. Hegelsche Philosophie wird oft als eine metaphysische Kons-truktion angesehen, in der einzelne Momente auf das Absolute hin teleologisch strukturiert werden. Demgegenüber ist die Rede von Hegelschem Holismus nicht nur unkontrovers, sondern beinahe selbstverständlich, weil «das Ganze» als grundlegender Begriff der Hegelschen Philosophie allgemein anerkannt wird. Nun möchte ich im 
folgenden nicht etwa versuchen, den ewigen Debatten um Hegelsche Selbstpositionierung ein Ende zu setzen. Ob Hegel seine Philosophie als den wahren telos, ja sogar als Ende der Ideen- und Kulturgeschichte verstanden hat und damit sich selber aus dem Strom des historischen Prozesses herausgehoben wissen wollte oder ob er sich doch bewusst war, dass auch seine Position nur «eigene Epoche in Gedanken» ausdrückt - diese ewigen Fragen der HegelForschung werden hier nicht entschieden. Es wird vielmehr eine moderate Position eingenommen, laut der Hegel, innerhalb seines holistischen Rahmens eine perspektivistische Vorgehensweise verwendet, um immer neue, immer konkretere, weil begrifflich immer mehr vermittelte, Einsichten in die kulturellen, sozialen und wissenschaftlichen Zusammenhängen zu erreichen. ${ }^{1}$ Ich werde im folgenden: (I) den von mir gemeinten Sinn der Termine »perspektivistisch « und »holistisch « im Kontext der Hegelschen Philosophie kurz erläutern; danach (II) werde ich die perspektivistische Darstellung des freien Willens bei Hegel in groben Zügen zu rekonstruieren versuchen; und schließlich werde ich (III) versuchen, aus der Auffassung der Willensfreiheit bei Hegel einige Schlüsse für die heutige Debatte über diesen Begriff zu ziehen.

Das Wort »Perspektive« wird von Hegel nicht als ein technischer Begriff verwendet; er selbst bevorzugt es, von den »Momenten«, »Verhältnissen« oder ihren »Seiten« zu sprechen. Nun hat aber die Übersetzung der Hegelschen Gedanken in das perspektivistische Vokabular viele wichtige Vorteile. Vor allem hilft es, das Hegelsche Konzept der Dialektik vor gravierenden Missverständnissen zu schützen. Durch die Hegelsche Rede von Momenten einer dialektischen Bewegung wurde eine Deutung nahe gelegt, welche die Synthese der Einseitigkeiten hervorhebt und damit in diese Momente eine teleologische Orientierung hineinschreibt. Das perspektivische Vokabular ermöglicht dagegen, solche Vorentscheidungen zu vermeiden und damit der ursprünglichen

1 Man könnte diese Provokation auch mit Bezug auf einflussreiche Namen und Theorien formulieren. Oft wird z.B. die Philosophie Friedrich Nietzsches als »perspektivistisch bezeichnet, was auch seinen Intentionen zu entsprechen scheint (etwa: »Wir, die DenkendEmpfindenden sind es, die wirklich und immerfort etwas machen, das noch nicht da ist: die ganze ewig wachsende Welt von Schätzungen, Farben, gewichten, Perspectiven, Stufenleitern, Bejahungen und Verneinungen«, F. Nietzsche, Die fröhliche Wissenschaft, Stuttgart 2000, S. 200 (§ 301)). Die perspektivistische Auffassung der Welt wird aber von Nietzsche selber als Gegensatz zu der kontemplativen Einstellung der bisherigen Philosophie gleichgesetzt, deren Höhepunkt oft im Hegelschen Denken gesehen wird. Deswegen meine ich, dass die Beziehung der Kategorie »Perspektivismus« auf Hegel in sich etwas Provokatives hat; dennoch glaube ich im folgenden zeigen zu können, dass dank der Anwendung dieser Kategorie eine zutreffende Beschreibung dessen, was Hegel unternimmt, gegeben werden kann. 
Hegelschen Intention gerecht zu bleiben, laut der die Dialektik kein »äußeres subjektives Denken« sein soll, das den Dingen aufgezwungen wird, sondern darin besteht, »eigene Arbeit der Vernunft der Sache zum Bewusstsein zu bringen «. ${ }^{2}$ Für eine solche Übersetzung spricht auch die Tatsache, dass Hegel selbst die Umgestaltung eines »an sich « in ein »für sich" nach dem Muster des Perspektivenwechsels konzipiert. Bei der Beschreibung einer jeweiligen, konkreten Weise des Subjekt-Objekt Verhältnisses betont Hegel sehr oft, dass einige Dimensionen dieses Verhältnisses nur für uns, Beobachter oder Zuschauer, zugänglich sind; im weiteren Verlauf geht es aber darum, dass sie für das Subjekt eines solchen Verhältnisses sichtbar werden sollen.

Viele Hegel-Interpreter würden zugeben, dass eine solche perspektivistische Vorgehensweise im Fall der Phänomenologie des Geistes von Hegel verwendet wird. Weil dort immer neue Zusammenhänge der Bewusstseinsgestalten und Wissensformen dargestellt werden, kann man das ohne weiteres als eine Reihe von »Paradigmenwechseln « bezeichnen. Hegelscher Perspektivismus zeigt sich hier ganz deutlich: es werden immer komplexere Auffassungen des SubjektObjekt Verhältnisses entfaltet. Jede neue Perspektive, welche den Anspruch auf Vollständigkeit erhebt, scheitert an eigener Unzulänglichkeit und verursacht eine Bewegung, sowohl auf der Seite des Bewusstseins, wie auch auf der Seite des Objekts (jede Bewusstseinsform hat mit einem neu strukturierten Objektkonzept zu tun). ${ }^{3}$ Ich möchte zeigen, dass diese perspektivistische Methode nicht nur bei der Transformation der Wissensformen in der Phänomenologie des Geistes, sondern auch in dem oft für konservativ gehaltenen und damit des absolutistisch-metaphysischen Dogmatismus sogar stärker bezichtigten späten Werk Hegels - in den Grundlinien der Philosophie des Rechts - erfolgreich verwendet wird. Um mich klar auszudrücken: ich glaube, Hegel verfährt auch hier perspektivistisch und sein Text wird viel verständlicher, wenn der Leser im Bezug auf ihn die perspektivistische Deutungsstrategie verwendet.

2 G.W.F. Hegel, Grundlinien der Philosophie des Rechts, Stuttgart 1995, S. 108 (§ 31).

3 So verweist etwa Ludwig Siep darauf, wie der Begriff der Erfahrung in der Phänomenologie zu verstehen sei: „Ihr wesentlicher Punkt ist, dass das »Für-es-Sein« des alten Gegenstandes (»Ansich«) zum neuen Gegenstand des Bewusstseins wird. Damit ist wohl gemeint, dass die Veränderungen, die eine Selbstkorrektur des Wissens in der zugrundeliegenden »Ontologie« (dem »Gegenstand«) hervorrufen, zu einer neuen Realitätsvorstellung führen, die Momente der alten und der veränderten Auffassung enthält« (L. Siep, Der Weg der Phänomenologie des Geistes. Ein einführender Kommentar zu Hegels "Differenzschrift« und »Phänomenologie des Geistes", Frankfurt am Main 2000, S. 77). Auch Thomas Sören Hoffmann spricht vom »gewaltigen Panorama von Bewusstseinsgestalten« in der Phänomenologie, wo »bestimmte Stellungen des Ichs zur Welt und zu den anderen « sich »als gewisse Idealtypik zeigen«; diese Stellungen folgen dabei »jeweils einer bestimmten, Subjekt wie Objekt formierenden Logik « (T.S. Hoffmann, Georg Wilhelm Friedrich Hegel. Eine Propädeutik, Wiesbaden 2004, S. 200). 
Die perspektivistische Methode Hegels möchte ich am Beispiel der Auffassung des Phänomens des freien Willens zeigen. Der erste Grund dafür ist, dass der freie Wille nicht etwa nur ein der wichtigen Begriffe der Grundlinien, sondern derjenige Begriff ist, welcher in diesen Vorlesungen aus verschiedenen Perspektiven aufgefasst und analysiert wird. Der freie Wille bildet die Grundlage des Rechts, der Moralität und der Sittlichkeit; er ist das Thema der Grundlinien. Ich werde mich also im folgenden auf das zentrale Thema dieses Werks konzentrieren; nicht alle Momente der komplexen Struktur des Textes können hier herausgearbeitet werden (ich werde mich nur auf Einleitung und auf Rechts- und Moralität-Kapiteln begrenzen, wo die grundlegenden Bestimmungen des freien Willens eingeführt und definiert werden).

Die methodologische Vorgehensweise Hegels läuft auf das Schema des Subjekts hinaus, welches immer wieder einen Schritt zurück macht, um seine vormalige Position aus einem neuen, breiteren Blickwinkel zu erfassen. Aus der neuen Perspektive erscheint die vormalige Auffassung einseitig, abstrakt und inhaltlich zu arm. Durch dieses konstante Abstandnehmen verlieren jedoch die Konturen des Bildes nicht an ihrer Schärfe; im Gegenteil: das Bild wird immer deutlicher, reicher und konkreter. Hegel geht ständig von einer einfachen Auffassung eines Phänomens aus, welcher Unzulänglichkeit zum Distanznehmen nötigt und eine neue, breitere und komplexere Auffassung generiert. Wichtig ist es hervorzuheben, dass nicht die jeweilige Auffassung der Sache, sondern der Vollständigkeits- oder Absolutheitsanspruch dieser jeweiligen Auffassung desavouiert wird. Aus der neuen Perspektive erscheint die vormalige nicht etwa als falsch, sondern als einseitig: sie behält ihre lokale, begrenzte Teil-Richtigkeit und wird aus der neuen Perspektiven auch immer mit-gesehen. Hier zeigt sich, nach meiner Auffassung, wie hilfreich die perspektivistische Deutung Hegels sein kann, etwa für das Verständnis dessen, was er unter seinem Begriff der "Aufhebung« meint. Dabei ist, wie schon angedeutet, die Frage, ob es eine absolute Perspektive gibt, aus der alle Teilperspektiven sichtbar werden, nicht die fundamentale Frage. In Grundlinien spricht Hegel ausdrücklich davon, dass nur das recht des Weltgeistes »das allerhöchste « ist. ${ }^{4}$ Die Perspektive des Weltgeistes ist aber eindeutig nicht die unsere; es wäre also sinnlos nachzudenken, ob wir sie annehmen dürfen oder können. Die Einführung dieser Perspektive sollte uns eher bewusst machen, dass es außer dem horizontalen Abstandnehmen auch vertikale Bewertungen der verschieden Perspektiven gibt, und zwar durch historische Zuordnungen. Hegel ordnet die Perspektiven in der Auffassung des freien Willens auch bestimmten Epochen des Geistes zu, was ihr Zusammenbestehen, also ihre Parallelität in der Gegenwart gar nicht ausschließt. Er kann auch blinde Flecken oder verlassene

4 G.W.F. Hegel, Grundlinien, S. 491 (§ 340). 
(falsche?) Perspektiven zeigen, aus denen etwas gerade nicht gesehen wurde wie zum Beispiel die Vernunftwidrigkeit der Sklaverei in der Antike.

Der zweite Grund meiner Themenwahl ist die große soziale Relevanz der aktuellen Debatte um den Begriff des freien Willens. Die Bedeutung dieser Debatte und ihres Ausganges geht eindeutig über die Grenzen des akademischen Diskurses hinaus und kann, wie Jürgen Habermas richtig eingesehen hat, gravierende Auswirkungen auf die Selbstdeutung unserer Gattung, auf die Kultur-, Gesellschafts- und Rechtsformen haben. ${ }^{5}$ Nun ist die aktuelle, zentrale Bedeutung der Debatte um den freien Willen aus der Perspektive Hegels keine Überraschung oder Zufall. Als Denker, der sich der Aufgabe stellt, das Vernünftige an dem Zeitgenössischen zu erkennen, sieht er das wahre Prinzip der modernen Welt in der Freiheit des Selbstbewusstseins. Insofern ist es aus Hegelschem Standpunkt nicht sehr verwunderlich, dass gerade um dieses Thema eine so heftige Debatte geführt wird. Es handelt sich aber nicht einfach um die Übereinstimmung in der Bewertung der Bedeutung dieser Fragen, sondern vor allem um die Art und Weise, auf welche Hegel diese Fragen thematisiert. Diese Vorgehensweise zeigt sich als sehr attraktiv und inspirierend, weil sie uns zu einer Distanznahme zu den bekannten Positionen in der aktuellen Debatte bewegen kann.

Die heutige Diskussion um Determinismus und Willensfreiheit zeichnet sich vor allem durch bipolare Aufteilungen: in Determinismen und Indeterminismen, Kompatibilismen und Inkompatibilismen, Naturalismen und Antireduktionismen. Es ist auffallend, dass sich Hegel in die so geteilten Kästchen nicht so richtig einordnen lässt. Ein Denker, der Freiheit als richtig verstandene Notwendigkeit deutet, ist gleichzeitig ein Denker, welcher Freiheit des Selbstbewusstseins für das Prinzip der modernen Welt hält. Die Vorgehensweise Hegels im Bezug auf den Begriff der Willensfreiheit zeigt sich, wie gesagt, als zugleich holistisch und perspektivistisch. Den ersten, holistischen Punkt, drückt Hegel auf folgende Weise aus:

»Dass der Wille frei und was Wille und Freiheit ist - die Deduktion hiervon kann (...) allein im Zusammenhange des Ganzen stattfinden ${ }^{6}{ }^{6}$

Dies bedeutet, dass es sich nicht auf einen Schlag erklären lässt, ob und in welchem Sinne der Wille frei ist. Zunächst wird, durch den Verweis auf die Tatsache des Bewusstseins, lediglich versichert, dass der Wille frei ist. Der

5 Indem Jürgen Habermas die Tragweite der naturalistischen Argumenten gegen Freiheit unter die Lupe nimmt, bemerkt er: »Dieser Determinismus ist allerdings mit dem alltäglichen Selbstverständnis handelnder Subjekte unvereinbar« (J. Habermas, Freiheit und Determinismus, in: J. Habermas, Zwischen Naturalismus und Religion. Philosophische Aufsätze, Frankfurt am Main 2005, S. 156).

6 G.W.F. Hegel, Grundlinien, S. 76 (§ 4). 
Beweis, der diese Versicherung begründen soll, wird von Hegel erst auf dem Weg der vielschichtigen philosophischen Deduktion geliefert. Dieser argumentative Leiter darf später nicht abgelegt werden, sobald man verstanden hat, worin die Freiheit des Willens besteht. Im Gegenteil: versucht man, aus den verschiedenen Kontexten, die die Freiheit des Willens zugleich manifestieren und ausmachen, den schlichten Satz »der Wille ist frei« herauszuheben und für sich zu reklamieren, dann wird nicht einfach etwas Falsches, sondern eher etwas Einseitiges und sehr Abstraktes behauptet. Somit ist der aktuell geführter Streit zwischen den Gehirnforschern und ihren philosophischen Kritikern nur ein der möglichen Bedeutungskontexte des freien Willens und darf als solcher nicht überbewertet oder sogar verabsolutiert werden.

\section{II}

Nun aber wollen wir die perspektivistische Darstellung des freien Willens in groben Zügen rekonstruieren. Im Zentrum des ersten Beschreibungsversuches dieses Phänomens steht die Möglichkeit des Abstrahierens:

»Der Wille enthält $\alpha$ ) das Element der reinen Unbestimmtheit oder der reinen Reflexion des Ich in sich, in welcher jede Beschränkung, jeder durch die Natur, die Bedürfnisse, Begierden und Triebe unmittelbar vorhandener oder, wodurch es sei, gegebener und bestimmter Inhalt aufgelöst ist; die schrankenlose Unendlichkeit der absoluten Abstraktion oder Allgemeinheit, das reine Denken seiner selbst (...). (...) absolute Möglichkeit von jeder Bestimmung, in der ich mich finde, oder die ich in mich gesetzt habe, abstrahieren zu können «.?

Von jedem Inhalt abstrahieren zu können setzt die Möglichkeit der Reflexion, also des Denkens voraus; somit wird schon in diesem ersten, rudimentären Moment auf die engste Verbindung des freien Willens und des Denkens verwiesen. Hegel wiederholt mehrmals, und zwar schon in den frühen Schriften, dass nur Wille als Intelligenz frei sein kann. Der fundamental negative Charakter des Willens, welcher in der Fähigkeit zum Ausdruck kommt, jede sinnliche Bestimmung in Klammern setzten zu können, davon Abstand zu nehmen, verweist auf den dem Wollen und dem Denken gemeinsamen Nenner der reflexiven Tätigkeit, der reflexiven »Unruhe«.

Die zweite von den fundamentalen Einsichten kann als komplementär zu der ersten gesehen werden und stellt fest, dass der Wille bei dieser Negation aller Inhalte nicht stehen bleiben kann, sondern sich eine »Besonderung «, eine konkrete Gestalt geben muss. Die Konkretisierung des Willens bildet nach diesem Modell ihre Positivierung im Sinne der Negation von Negation:

7 Ebd., S. 77 (§ 5).

Suplemento 19 (2014) de Contrastes. Revista internacional de filosofía 
»Ebenso ist Ich das Übergehen aus unterschiedsloser Unbestimmtheit zur Unterscheidung, Bestimmen und Setzen einer Bestimmtheit als eines Inhalts und Gegenstands. (...) Dies zweite Moment der Bestimmung ist ebenso Negativität, Aufheben als das erste - es ist nämlich das Aufheben der ersten abstrakten Negativität $\ll^{8}$

Diese philosophischen Vorentscheidungen sind sehr wichtig. Hegel könnte viel einfacher verfahren und den Willen als Abstraktion von dem vorgegebenen Material konzipieren. Dass er aber diesen Weg nicht wählt und von dem Abstrahierenkönnen ausgeht, um erst im zweiten Schritt das Positive - den Gegenstand des Wollens - als Negation der ersten Negativität des Willens deutet, hat einen entscheidenden Sinn. So konzipiert, muss sich nämlich der Wille nicht erst aus dem Sinnlichen herausheben, sondern er gibt sich gewissermaßen seine Sinnlichkeit selbst. Schon bei Johann Gottlieb Fichte werden etwa die menschlichen Triebe nicht vorgefunden, sondern durch das Ich gesetzt. Fichte, ebenso wie später Hegel, scheint hier der entscheidenden Einsicht Immanuel Kants zu folgen, wonach ein Vernunftwesen auch dann frei handelt, wenn es durch Triebe und Begierden zu seiner Tat bestimmt wird, weil es selbst die Triebe, Begierden oder Neigungen als Grundlage dieser Tat akzeptiert (Kantisch gesprochen: »in seine Maxime aufgenommen «) hat. ${ }^{10}$ Hegel ist sich offensichtlich auch bewusst, dass er, sobald er die naturalistische Annahme akzeptieren würde, laut welcher der Wille nur raffinierte Gestalt des natürlichen Triebes sei, auch niemals zu dem eigentlichen Begriff des freien Willens gelangen würde, welcher sich selber eine Wirklichkeit, als den Bereich seiner Anwendung, gibt.

Nach der zweiten Einsicht muss also Wille immer eine Objektivität haben, auch wenn später diese Objektivität nicht mehr eine natürliche, sondern eine aus dem Geiste hervorgebrachte sein wird. Nun würden die beiden Momente des Willens voneinander getrennt nur einseitig und abstrakt bleiben; der Wille besteht gerade in der »Einheit dieser beiden Momente $« .{ }^{11}$ Der Wille ist »die in sich reflektierte und dadurch zur Allgemeinheit zurückgeführte Besonderheit; Einzelnheit; die Selbstbestimmung des Ich in einem, sich als das Negative seiner selbst, nämlich als bestimmt, beschränkt zu setzten und bei sich, d.i. in seiner

8 Ebd., S. 79 (§ 6).

9 Siehe vor allem: J.G. Fichte, Das System der Sittenlehre nach den Prinzipien der Wissenschaftslehre (1798), Hamburg 1995 (»Ein Trieb aber, der als wesentlich, bestehend, unaustilgbar gesetzt wird, treibt; und dieses ist seine Äußerung«, J.G. Fichte, Das System der Sittenlehre, S. 39-40).

10 I. Kant, Die Religion innerhalb der Grenzen der blossen Vernunft, in: I. Kant, Werke in sechs Bänden, W. Weischedel (Hrsg.), Darmstadt 1998, B 12.

11 G.W.F. Hegel, Grundlinien, S. 82 (§ 7). 
Identität mit sich und Allgemeinheit, zu bleiben (...) «. ${ }^{12}$ Der freie Wille wird hier also als Einheit der Allgemeinheit und der Besonderheit, der Negation aller Inhalte und des Sich-Inhalt-Gebens verstanden. Wille ist immer als Einheit der Fremdreferenz und Selbstreferenz zu verstehen. Diese Grundstruktur des Willens wird in jeder weiteren Ansicht der Freiheit immer mit-gesehen. Auf jeder Stufe der Verwirklichung der Freiheit wird die Fähigkeit des Willens, sich Inhalt zu geben und auf ihn Bezug zu nehmen, durch seine andere Grundfähigkeit begleitet, von jedem solchen Inhalt auch abstrahieren zu können.

Dieser erste Anlauf in der Rekonstruktion des freien Willens kann zu nichts anderes gelangen als zu einem »natürlichen und unmittelbaren« Willen, also zu der Instanz, die in der philosophischen Tradition als liberum arbitrium und in der deutschen Philosophie als Willkür bezeichnet wird. Der natürliche und unmittelbare Wille ist als Möglichkeit der Wahl zu verstehen, wobei sich diese Wahl auf die natürlichen Triebe, Bedürfnisse und Gegenstände, die ihrer Befriedigung dienen, bezieht. Schon auf dieser ersten Stufe, oder schon aus dieser ersten, noch undifferenzierten Perspektive, ist Wille (als Willkür) frei zu nennen. Hegel versteht die Freiheit der Willkür als die rudimentäre (immer noch abstrakte, unentwickelte und einseitige) Form der Freiheit. Aber schon auf dieser Stufe wird die Distanz zu dem Bereich der Natur immer größer. Die Triebe, Begierden und Neigungen werden nicht einfach als gegeben betrachtet, sondern allmählich gereinigt: sie werden reflexiv vorgestellt, verglichen und systematisiert. Dadurch verlieren sie ihre natürliche Rohheit. Hegel führt in diesem Zusammenhang den Begriff der Bildung ein. Diese Einsicht kann man auch so formulieren, dass die Freiheit, schon in dieser ersten, einfachen Auffassung, als Wahl zwischen Optionen, einen gewissen Grad der kulturellen Entwicklung impliziert und sich nur in der Umgebung einer solchen kulturellen Entwicklung konstituieren und entfalten kann.

Freiheit des Willens als Willkür, als Möglichkeit der Wahl, ist in der Konstruktion Hegels eine Unmittelbarkeit, welche die Form des abstrakten Rechts impliziert. Die Selbstreferenz des einzelnen, besonderen Willen ist hier noch abstrakt und führt lediglich zu dem Konzept der (rechtlichen) Persönlichkeit. »Das Rechtsgebot ist daher: sei eine Person und respektiere die andern als Personen « ${ }^{13}$ Unter dem Begriff der Persönlichkeit wird lediglich die Fähigkeit - die eigene Fähigkeit und die Fähigkeit der Anderen - gemeint, sich selber und äußere Gegenstände in Besitz zu nehmen und gleichzeitig von jedem Inhalte (Besitz, Eigentum), zu dem die Person berechtigt ist, abstrahieren zu können. Indem ich die anderen als Personen anerkenne, räume ich ihnen zunächst und vor allem das Recht des Besitzes und der Verfügung über die Sachen, aber auch über ihren eigenen Körper ein. »Die Person muss sich eine

12 Ebd.

13 Ebd., S. 118 (§ 36). 
äußere Sphäre ihrer Freiheit geben, um als Idee zu sein «. ${ }^{14}$ Diese äußere Sphäre der Freiheit wird durch das Eigentum errichtet, das hier nicht vor allem als Befriedigung der materiellen Bedürfnisse, sondern in erster Linie als Manifestation der Freiheit gedeutet wird.

»Eigentum zu haben erscheint in Rücksicht auf das Bedürfnis, in dem dieses zum Ersten gemacht wird, als Mittel; die wahrhafte Stellung aber ist, dass vom Standpunkte der Freiheit aus das Eigentum als das erste Dasein derselben wesentlicher Zweck für sich ist «. ${ }^{15}$

»Die Person hat das Recht, in jede Sache ihren Willen zu legen, welche dadurch die Meinige ist, zu ihrem substantiellen Zwecke, das sie einen solchen nicht in sich hat, ihre Bestimmung und Seele meinen Willen erhält - absolutes Zug-eignungsrecht des Menschen auf alle Sachen «. ${ }^{16}$

Freiheit auf der ersten Stufe bedeutet also die Umgestaltung des natürlichen Daseins der Dinge in die von unserem Willen abhängige Sachen. Dies betrifft nicht nur äußere Sachen, sondern auch unsere veräußerten Talente und Fähigkeiten, ja unseren eigenen Leib. Als (rechtliche) Person bin ich nicht nur Besitzer der Sachen, sondern auch meines eigenen Körpers, mitsamt seiner physischen und geistlichen Fähigkeiten. Indem ich mich auf andere Personen beziehe, die ebenso das Verfügungsrecht über ihre Körper und ihre Sachen haben, trete ich mit ihnen in die durch das Recht geregelten Verhältnisse. Alle diese Verhältnisse werden unter dem Begriff des Vertrags subsumiert. Der Vertrag zeichnet sich durch reine Äußerlichkeit aus; die Willen der Vertragsparteien beziehen sich aufeinander in rein negativer Weise. Es geht um die Achtung der ausgegrenzten Eigentumssphären und um das Verbot ihrer Übertretung.

Das erste, grundlegende Konzept der Willensfreiheit als Willkür - als Möglichkeit der Wahl - impliziert also einen ganzen Zusammenhang der Ver-hältnisse und Begriffe, in deren Kontext die Idee des freien Willens ihre erste Bedeutung gewinnt. Diese Begriffe sind: Person, Besitz, Eigentum, Recht, Vertrag. Auf der Stufe, auf welcher das Subjektive in der Verwirklichung der Freiheit den Status der Person erreicht, wird die Realität gewissermaßen als allgemeines Substrat des Privatbesitzes der Personen betrachtet. Auf dieser Stufe bedeutet Freiheit also den allgemeinen Anspruch der einzelnen Personen auf Besitz und Verfügung über die Objekte der Welt und über eigenen, objektivierten Leib. Das Materielle, sowohl der äußeren Sachen, wie auch des eigenen Körpers, wird angeeignet. Der Akt der universalen Aneignung setzt die ganze ma-

14 Ebd., S. 123 (§ 41).

15 Ebd., S. 128 (§ 45).

16 Ebd., S. 126-127 (§ 44).

Suplemento 19 (2014) de Contrastes. Revista internacional de filosofía 
terielle Wirklichkeit in Klammern: der abstrakte freie Wille macht sich die Welt zu eigen.

Nun wird aber, in dem nächsten Schritt, die Unvollständigkeit eines solchen Bildes der Freiheit konstatiert; ein reicheres Bild soll aus der neuen Perspektive entworfen werden. Es ist die Perspektive der moralischen Freiheit. War die vorherige Stufe vornehmlich nach Außen gerichtet, so wird die moralische Perspektive den Akzent auf die Selbstreferenz des Einzelnen setzen. Der Wille muss aber zunächst in sich selber zurückgreifen und eine neue Stufe der Reflexivität erringen. Durch die Veränderung des Standpunktes wird das Merkmal des Willens: seine Unendlichkeit, die bisher nur durch uns (die Beobachter) gesehen wurde, dem Willen selbst bewusst (der Wille wird jetzt für sich unendlich). Der Beobachter nimmt nicht nur einen Abstand, sondern verändert den Blickpunkt; diesmal richtet er sich mehr auf den subjektiven, als auf den objektiven Pol. Diese neue Ansicht drückt sich in der qualitativ neuen Art der Selbstreferenz aus: in der Selbstbestimmung (Freiheit als Autonomie). Der Wille bestimmt jetzt sich selber zu einer Tat; er verpflichtet sich selber bewusst, etwas zu unternehmen oder zu unterlassen.

»Beim strengen Recht kam es nicht darauf an, was mein Grundsatz oder meine Absicht war. Diese Frage nach der Selbstbestimmung und Triebfeder des Willens, wie nach dem Vorsatz, tritt hier nun beim Moralischen ein. Indem der Mensch nach seiner Selbstbestimmung beurteilt sein will, ist er in dieser Beziehung frei $(\ldots){ }^{17}{ }^{17}$

Es wird nur dasjenige als eigenes Tun anerkannt, was aus dem Bewusstsein als Absicht des Subjekts fließt und was vor dem Tribunal des Bewusstseins geprüft und akzeptiert wurde. War in der vormaligen Auffassung der Wille als Willkür aufgefasst worden, so ist hier offensichtlich der vernünftige Wille als Ausdruck der Autonomie gemeint. Das Kantische Moment der Autonomie wird dabei einerseits hervorgehoben und für absolut anerkannt, andererseits jedoch bleibt hier die innerliche Selbstbestimmung notwendigerweise gegenstandslos:

»Der moralische Standpunkt ist daher in seiner Gestalt das Recht des subjektiven Willens. Nach diesem Rechte anerkennt und ist der Wille nur etwas insofern es das Seinige, er darin sich als Subjektives ist. (...) [Gleichzeitig jedoch] Das Selbstbestimmen ist in der Moralität als die reine Unruhe und Tätigkeit zu denken, die noch zu keinem was ist kommen kann «. ${ }^{18}$

17 Ebd., S. 212 (§ 106 Zusatz).

18 Ebd., S. 213-214 (§ 107, § 108 Zusatz). 
Durch die Fixierung des Autonomiekonzeptes auf dem subjektiven Selbstbezug wird er paradoxerweise gerade dem äußeren, sinnlichen Material, das seinen Inhalt ausmachen muss, preisgegeben. ${ }^{19}$ Dies zeigt sich vor allem in der Inhaltslosigkeit des kategorischen Imperativs, als dem höchsten Ausdrucks der subjektiven Autonomie. Was die Pflichten sind, soll sich aus dem formalen Selbstbezug des subjektiven Bewusstseins ergeben; und doch ist es von dem Vorhandensein bestimmter Praktiken und Institutionen abhängig:

»Wenn wir auch oben den Standpunkt der Kantischen Philosophie hervorhoben, der, insofern er das Gemäßsein der Pflicht mit der Vernunft aufstellt, ein erhabener ist, so muss doch hier der Mangel aufgedeckt werden, dass diesem Standpunkte alle Gliederung fehlt. Denn der Satz: Betrachte, ob deine Maxime könne als ein allgemeiner Grundsatz aufgestellt werden, wäre sehr gut, wenn wir schon bestimmte Prinzipien über das hätten, was zu tun sei. Indem wir nämlich von einem Prinzipe verlangen, es solle auch Bestimmung einer allgemeinen Gesetzgebung sein können, so setzt eine solche einen Inhalt schon voraus $(\ldots) \ll^{20}$

Also schon in dem entscheidenden Zug dieser neuen Ansicht der Willensfreiheit wird angedeutet, warum diese Ansicht nur begrenzt und unvollständig bleiben muss; warum sie - anders gesagt - nicht den ganzen Anwendungsbereich des Willensbegriffs klar überblicken lässt. Mit dem Autonomiekonzept erreicht jedoch der Wille eine neue Dimension: es ist nicht mehr Wille als Wahl zwischen den vorgegebenen Optionen, nicht mehr Wille, der diese oder jene äußere Gegenstände will, sondern es ist »der Wille, der den Willen selbst will«. Erst auf diesem selbstreferenziellen - durch die Vorstellung des Gewollten und durch die bewusste Akzeptanz des Gewollten vermittelten Niveau können sich die Standpunkte der Moral und der Sittlichkeit entwickeln. Einerseits wird also, im Vergleich mit der vorherigen Perspektive des Wollens als Willkür, der Wille mehr autonom, mehr unabhängig von den äußeren Umständen und Objekten, und damit mehr subjektiv (selbstreferenziell). Gleichzeitig aber, wie in der ersten, einfacheren Auffassung des Wollens als Willkür, so bildet sich auch hier, im Rahmen der Auffassung des Willens als der Autonomie des Subjekts, ein neues Konzept der Realität. Es ist nicht mehr Realität

19 Schon der junge Hegel verwendet dieses kritische Argument, indem er etwa in Glauben und Wissen mit Bezug auf das Denken Kants, Fichtes und Jacobis folgenden Vorwurf erhebt: »(...) Weil diese Vernunft schlechthin nur diese Richtung gegen das Empirische hat, das Unendliche an sich nur in Beziehung auf das Endliche ist, so sind diese Philosophien, indem sie das Empirische bekämpfen, unmittelbar in seiner Sphäre geblieben (...)« (G.W.F. Hegel, Glauben und Wissen oder die Reflexionsphilosophie der Subjektivität in der Vollständigkeit ihrer Formen als Kantische, Jacobische und Fichtesche Philosophie, in: G.W.F. Hegel, Jenaer Schriften 1801-1807, Frankfurt am Main 1996, S. 296).

20 G.W.F. Hegel, Grundlinien, S. 254 (§ 135 Zusatz). 
als statischer Bereich des potentiellen Besitznehmens, des Zueignens, sondern die Realität wird jetzt aufgefasst als dynamischer Bereich der Zu-VeränderndenWelt, oder besser: der Zu-Beiflussenden-Weltlauf.

Mit der Veränderung des Willens- und des Gegenstandkonzeptes geht auch eine wichtige Differenzierung ihrer Relation einher. Aus der Handlung wird eine Tat. Zu einer Handlung, deren Begriff, wohlgemerkt, für den Bereich des abstrakten Rechts ausreichte, wäre auch ein Tier fähig. Zu einer Tat ist dagegen nur der Mensch fähig, weil er unterscheiden kann, was in seiner Absicht lag und was sich der äußeren Notwendigkeiten ergab und nicht ihm zuzurechnen ist. Absicht ist dabei von Hegel als eine allgemeine Auffassung der geplanten Handlung verstanden, im Gegensatz zu einem bloßen Vorsatz. Auf klare Weise unterscheidet dies Hegel im folgenden Zitat: »Blödsinnige, Kinder wissen wohl, dass sie zuschlagen, brennen, aber nicht, dass sie damit töten, töten können - diese Möglichkeit ist das Allgemeine der Handlung [Absicht - J. K.-K.] - Im Vorsatze kann nur gelegen haben, zuzuschlagen ${ }^{21}$.

Nach dieser Unterscheidung ist also dem handelnden Einzelnen nur dasjenige zuzuschreiben, was in seiner Absicht lag. Hegel ist sich dabei völlig bewusst, dass eine klare Demarkationslinie zwischen dem, was in Absicht lag und anderen Umständen fast nie eindeutig zu ziehen ist: dies ist auch einer der Gründe, aus welchen Hegel sowohl die Kantisch gesinnte Deontologie, wie auch den entgegengesetzten, radikalen Konsequentialismus in der Ethik gleichermaßen ablehnt. »Der Grundsatz: bei den Handlungen die Konsequenzen verachten, und der andere: die Handlungen aus den Folgen beurteilen und sie zum Maßstabe dessen, was recht und gut sei, zu machen - ist beides gleich abstrakter Verstand $\kappa^{22}$ Eindeutig perspektivistischer Natur ist die Überlegung, dass in einem anderen, historischen oder kulturellen, Kontext die Tat ganz anders angesehen werden kann: wo man die Unterscheidung zwischen dem Vorsatz und der Absicht auf der einen Seite, und zwischen dem bewusst Akzeptierten und dem der Handlung Zufälligen auf der anderen nicht durchführt - wie etwa in der Antike - gelangt man zu einer völlig anderen, tragischen Auffassung der menschlichen Tat. Im Rahmen dieser Auffassung wird der Begriff der Schuld nicht subjektiv, sondern objektiv verstanden:

»Jedes einzelne Moment, das sich als Bedingung, Grund, Ursache eines solchen Umstandes zeigt und somit das Seinige beigetragen hat, kann angesehen werden, dass er schuld daran sei oder wenigstens schuld daran habe«. ${ }^{23}$

21 Ebd., S. 231 (§ 120).

22 Ebd., S. 224 (§ 118).

23 Ebd., S. $222(\S 115)$ 
Gerade weil die antike Ansicht der Tat den Vorsatz von der Absicht (und, parallel dazu, die bewusst akzeptierten von den zufälligen Folgen) nicht unterscheidet, nimmt der tragische Held die ganze Schuld auf sich. Die subjektive Auffassung der Tat verliert diese »Gediegenheit« und drückt die Forderung aus, nur für die bewusst anerkannten Folgen der Handlung Verantwortung zu übernehmen. Einer der Gründe, warum Hegel die verlorene oder nicht mehr eingenommene antike Perspektive in der Auffassung der Tat überhaupt berücksichtigt, ist es, dem philosophischen Beobachter klar zu machen, dass das subjektive Moment an der Handlung nicht eine Selbstverständlichkeit ist, also nicht aus jeder Perspektive sichtbar wäre. Die Steigerung des Subjektiven in der Bewertung der Handlung und ihrer Konsequenzen gehört zum Entwurf der Moderne.

Diese Steigerung der Subjektivität manifestiert sich allerdings auch in Veränderung der Handlungsmotivation des Willens: die Handlungen, die in dem Willen wurzeln, sollen nicht nur dem Recht entsprechen, sie sollen auch dem Wohl des Einzelnen und Aller dienen. »Das Wohl hat in dieser Idee [des Guten - J. K.-K.] keine Gültigkeit für sich als Dasein des einzelnen besonderen Willens, sondern nur als allgemeines Wohl (...) - das Wohl ist nicht ein Gutes ohne das Recht. Ebenso ist das Recht nicht das Gute ohne das Wohl (fiat iustitia soll nicht pereat mundus zur Folge haben) «. ${ }^{24}$ Die Handlungen des freien Willens sollen nicht nur auf die formale Übereinstimmung mit den Prinzipien des Rechts, welche die Eigentumssphären der Einzelnen negativ sichert, sondern auf das Wohl - mein und das allgemeine - positiv bezogen werden. War in der ersten Perspektive Recht, so ist hier Recht und Wohl als Ziel der Handlung gesehen. Aus dieser Verbindung von Recht und Wohl resultiert die Idee des Guten, die reicher ist, als nur das formale, abstrakte Recht. Allerdings zeigt sich diese Idee des Guten hier immer noch zu abstrakt, weil sein Konzept und seine Verwirklichung dem subjektiven Willen und dem Gewissen überlassen bleibt.

Das Gewissen, als die Instanz, die für die Verwirklichung des Guten zuständig sein soll, ist für Hegel die Quelle der Pflichten. Darin, dass die innere Instanz des Gewissens zur Grundlage und Maßstab der Pflichten avanciert, manifestiert sich die moderne Hervorhebung der Subjektivität, die als gültig nur das anerkennen kann, was vor ihrem Tribunal stand hält und von ihr frei und bewusst anerkannt wird. Deswegen ist die »Entdeckung « des Gewissens und ihre Reinigung von allen empirischen, heteronomen Momenten, welche durch die Kantische Philosophie geleistet worden ist, eine unwiderrufliche Errungenschaft der Moderne. »Der Mensch ist als Gewissen von den Zwecken der Besonderheit nicht mehr gefesselt, und dieses ist somit ein hoher Standpunkt, ein Standpunkt der modernen Welt $(\ldots) \ll .{ }^{25}$ Gleichzeitig verliert sich ein stark

24 Ebd., S. 245 (§ 130).

25 Ebd., S. 254-255 (§ 136 Zusatz).

Suplemento 19 (2014) de Contrastes. Revista internacional de filosofía 
immanentiesiertes Gewissen in seinen guten Absichten, die eine abstrakt formulierte Idee des Guten zu realisieren trachten; da die Idee des Guten zunächst nur subjektiv ist und in den sozial anerkannten, allgemeinen Institutionen den $\mathrm{Fu} ß$ noch nicht gefasst hat, muss auch das an die Realisierung des Guten interessierte Gewissen einseitig und abstrakt bleiben. Hegel for-muliert hier das Paradoxon des Gewissens, welches er folgendermaßen beschreibt:

»Die Zweideutigkeit in Ansehung des Gewissens liegt daher darin, dass es in der Bedeutung jener Identität des subjektiven Wissens und Wollens und des wahrhaften Guten vorausgesetzt und so als ein Heiliges behauptet und anerkannt wird und ebenso als die nur subjektive Reflexion des Selbstbewusstseins in sich, doch auf die Berechtigung Anspruch macht, welche jener Identität selbst nur vermöge ihres an und für sich gültigen vernünftigen Inhalts zukommt «. ${ }^{26}$

Das Paradoxon besteht also offensichtlich darin, dass die an sich gültige und für die Moderne enorm wichtige, objektive Idee des Gewissens nur durch das individuelle Gewissen instantiiert werden kann, dass aber gleichzeitig das individuelle Gewissen aus prinzipiellen Gründen dieser objektiven Idee nicht entsprechen kann. Was das individuelle Gewissen für das Gute - also vornehmlich was es in moralischer Hinsicht für das Wohl der Anderen - hält, kann deswegen sowohl mit den Überzeugungen anderer, wie auch mit dem Ge-setz sehr schnell in Widerspruch geraten. Ludwig Siep hat in diesem Kontext das erläuternde Beispiel der Gewissenstätern benutzt, die gegen das Gesetz, aber aus der Tiefe des eigenen Gewissens und mit der guten, von der Vorstellung der Wohl der Anderen geleiteten, Absicht handeln. ${ }^{27}$ Die Tatsache, dass Hegel dieses für die moderne Gesellschaft typische Phänomen auch mitgemeint hat, wird deutlich in dem folgenden Zitat, in welchem Hegel das Gewissen als potentielle Grundlage der gesellschaftlichen Ordnung mit klaren Worten ablehnt: »Wenn ich vorauszusetzen hätte, sie [die Menschen - J. K.-K.] wären irrende Gewissen, anerkennten nur das für Recht, was sie nur in ihrem subjektiven Gewissen fänden, so dass sie darin das Gegenteil von allem, was recht und sittlich, fänden, so befände ich mich ärger als unter Räubern $(. ..) \ll{ }^{28}$ Das individuelle Gewissen, zur Grundlage der Pflichten und zum Maßstab der Beurteilung der Handlungsabsichten gemacht, bringt unumgänglich die Gefahr der »Verflüchtigung aller Momente« mit sich und wird dadurch, als Entscheidungsinstanz, mit dem Risiko des irrationalen Dezisionismus geplagt.

Die weitere Konstruktion der Grundlinien zeigt dann, dass aus diesem Punkt notwendigerweise zum Guten als etwas In-der-Welt-Realisierten übergegangen

26 Ebd., S. 256 (§ 137).

27 Während seines Vortrags an der Universität Warschau, am 09.04.2010.

28 G.W.F. Hegel, Grundlinien, S. 258 (§ 137). 
werden muss. Dieser Übergang ist der Inhalt des ganzen Sittlichkeit-Teils der Vorlesungen, welches hier nicht mehr analysiert werden kann. Das Gute wird im aufeinanderfolgenden sozialen Rahmen der Familie, der bürgerlichen Gesellschaft und, schließlich, des Staates, objektiviert. Erst hier, in der Sphäre der ausdifferenzierten Sittlichkeit, fühlt sich das moderne Individuum sowohl in seinem Inneren, wie auch in seinem Äußeren zu Hause. Das Recht, das in dieser Sphäre nicht mehr ausschließlich Eigentumsverhältnisse, sondern die hochkomplexen Relationen innerhalb der Gesellschaft und Staat reguliert, nimmt vom Individuums Schultern die Last des individuellen Entscheidens und die damit zusammenhängende Unsicherheit, die immer mit dem Ausgang der moralischen Reflexion verbunden ist. Dies bedeutet jedoch keinesfalls, dass diese neue inzwischen schon dritte - Perspektive, aus welcher Willensfreiheit aufgefasst wird, die vormalige Bedeutung des Gewissens aus dem Auge verliert. Im Gegenteil: Hegel weist in seiner Beschreibung der bürgerlichen Gesellschaft auf verschiedene Momente hin, wo die Anwendung der individuellen moralischen Perspektive durch soziale Leistungen nie völlig verdrängt werden kann - wie es etwa im Bereich der sozialen Hilfe der Fall ist.

Wichtig ist, dass in der Sphäre der Moralität, mit der neuen Ansicht des freien Willens auch sein neuer begrifflicher Zusammenhang von Hegel eingeführt wurde: diesmal waren es solche Begriffe, wie: Subjekt, Vorsatz, Absicht, Tat, Weltverlauf, Schuld, Gewissen, das Gute, das Wohl, die Pflicht. Um es nochmals zu unterstreichen: einer neuen Auffassung des Trägers der Willensfreiheit entspricht immer ein neues Konzept des Gegenstandsbereiches: der Person - rechtlose Sachen als ihr potentielles Eigentum; dem Subjekt - die Welt, als Sphäre der bewussten Tat; dem Menschen - das System der Bedürfnisse innerhalb der bürgerlichen Gesellschaft; dem Bürger - die soziale Welt (die sich in verschiedenen gesellschaftlichen und staatlichen Praktiken und Institutionen manifestiert). Erst alle diese Perspektiven oder Paradigmen zusammen machen den komplexen Inhalt des Begriffes der Willensfreiheit aus oder, mehr im Sinne Hegels formuliert: sie lassen dem Begriff der Willensfreiheit sich völlig entfalten.

\section{III}

Versuchen wir jetzt aus der nur in groben Zügen dargestellten perspektivistischen und holistischen Auffassung der Willensfreiheit bei Hegel einige Schlüsse für die heutige Debatte um diesen Begriff zu ziehen. Wir sehen sogleich, dass aus einer durch Hegel inspirierten Perspektive vor allem die unreflektierte Wahl der Kriterien fraglich erscheint, nach welchen das Problem des freien Willens heute beurteilt werden soll. Die Frage »Ob der Wille wirklich frei ist«, oder präziser gesagt: »Ob der Mensch seine Entscheidungen aus Freiheit oder aus Notwendigkeit trifft«, ist nicht einfach ungültig; eine wahrhaft 
perspektivistische und holistische Philosophie soll aber diese Frage nicht übereilig zu beantworten versuchen, sondern vielmehr darüber kritisch nachdenken, auf welche Weise eine solche Frage zu beantworten wäre. Zum ersten: warum soll hier nur die Frage der Willensfreiheit des Subjekts beantwortet werden? Warum wird die Frage der Willensfreiheit der Person, des Menschen und des Bürgers vernachlässigt? Was nötigt uns, nur die subjektiv-psychologische Perspektive zu bevorzugen? Und sollten wir uns schon auf diese enge Perspektive begrenzen, wieso wird nicht reflektiert, wie wir mit diesem unvollständigen, aber dennoch komplexen Begriff einer subjektiven Freiheit, vorgehen sollen? Sollen hierzu: Experimente durchgeführt werden? Lässt sich also der freie Wille nachweisen oder, umgekehrt, empirisch falsifizieren? Oder ist bei der Beantwortung dieser Frage eher eine diskursive Vorgehensweise notwendig? Soll die Willensfreiheit als eine psychologisch-pragmatische Angelegenheit oder vielleicht als rechtliches, kulturelles, moralisches, ökonomisches, politisches und ethisches Phänomen gedeutet werden? Aus welchem Grund sollte uns erlaubt werden, den pszchologischen Begriff der Willensfreiheit aus dem ganzen Gefüge von Begriffen (Person, Subjekt, Mensch, Bürger, Recht, Vorsatz, Absicht, das Gute, Pflicht, das Wohl usw. usf.) herauszureißen und einen solchen dekontextualisierten Begriff wie ein empirisches, greifbares Phänomen zu betrachten, welches sich in den Laboratorien entweder beglaubigen oder falsifizieren ließe?

Nun stellt sich hier leicht ein Gegenvorwurf ein: die soeben gestellten kritischen Fragen könnten durch den Naturalisten als eine Art »courtier's reply« gesehen werden. Seine Antwort auf unseren Einwand, er vergesse die komplexen Zusammenhänge, in die der Begriff der individuellen Willensfreiheit eingebaut ist, könnte folgendermaßen lauten: Wie wäre es, wenn wir gerade in einen Zeitalter eintreten, wo der Begriff des freien Willens nicht mehr nötig ist; wir wissen, dass damit notwendige Veränderungen im ganzen sozialen Netz der Praktiken und Institutionen verbunden sind. Es könnte doch sein, dass angesichts der schlagenden Argumente der Gehirnforscher, das ganze Netz der um die somit falsifizierte »Bewusstseinstatsache« der Freiheit aufgebauten Überzeugungen, Praktiken und Institutionen verändert werden muss. Wäre aber ein solcher kohärenter Paradigmenwechsel in neuer Epoche vorstellbar und durchführbar, bliebe einem hegelschen Denker nichts anderes, als diese neue Epoche »in Gedanken« zu fassen. Er konnte sie jedenfalls nicht aus der Perspektive des »perennierenden Sollens « kritisieren.

Vor einem solchen Vorwurf könnte man den Hegelschen Standpunkt verteidigen, indem man auf die Wittgensteinsche Unterscheidung zurückgreift zwischen den verschiedenen Momenten unseres Überzeugungsnetzes. ${ }^{29}$ Es gibt solche Überzeugungen, die wir leicht dem Zweifel preisgeben, weil sie nur am

29 Siehe: L. Wittgenstein: Über Gewißheit, Frankfurt am Main 1984. 
Rande unseres Überzeugungsnetzes stehen; es gibt aber auch den harten Kern, den wir nur sehr ungern antasten. Zu diesem Kern würde auch die Idee der Willensfreiheit gehören. Wir müssten zu viel im Netz der sozialen und rechtlichen Institutionen und Verhältnissen verändern, wenn wir des freien Willens los werden wollten; der freie Wille steht uns gleichsam nicht zur Verfügung, er liegt als Begriff zu zentral in dem Diskurs der Moderne, als dass wir ihn daraus herausheben und über Bord werfen könnten. ${ }^{30}$ Dies wäre vielleicht eine verlockende Lesart; der Hegelschen Philosophie wäre sie aber nicht gerecht. Sie mutet zu pragmatisch an: es ist zwar wahr, dass Hegel die verschiedenen Epochen nicht moralisch beurteilt. Deswegen sagt er nicht einfach unvermittelt, dass die Freiheit der Alten etwas Schlechtes, die Freiheit der Modernen dagegen etwas Gutes sei. Gleichzeitig scheint er jedoch die moderne Vorstellung der Freiheit des Selbstbewusstseins doch zu einem allgemeinen Kriterium zu stilisieren; ansonsten könnte er nicht mit einer solchen Entschiedenheit den Sklavenstatus der Kinder im römischen Recht ablehnen oder Platon für den Ausschluss des Privateigentums kritisieren.

Deshalb scheint es mir viel erfolgversprechender, und der Intention Hegels mehr gerecht, wenn die Polemik mit den Naturalisten eher auf die weder historisch noch kulturell oder sozial relativierbare Verbindung des Denkens und des freien Willens gestützt wird. Denken und Wollen bilden für Hegel eindeutig zwei Seiten derselben Medaille. Das Denken wird von Hegel immer als Aktivität konzipiert, und zwar sowohl auf individueller, wie auch auf objektiver Ebene: der Begriff ist für Hegel nie etwas Statisches, im voraus Gegebenes, es ist immer entelechia, es bewegt sich, entwickelt sich, um zur vollkommenen Gestalt zu kommen. Ähnliches gilt aber auch umgekehrt: Praxis, sowohl die individuelle, wie auch die objektive Praxis des Begriffs (Arbeit des Begriffs, wie das Hegel so oft nennt) ist teleologisch konzipiert, das heißt, sie bedeutet vor allem Verwirklichung der Zwecke - und Zwecke können nur bewusst gesetzt werden, sie implizieren also (objektiv) Begrifflichkeit und (subjektiv) Kognition. Sobald die dogmatische Unterscheidung in die begriffliche Welt des Denkens und außerbegriffliche Welt der Sachen (und die dogmatische Gegenüberstellung von Denken und Wollen, von Theorie und Praxis) unterlaufen wird, kann der freie Wille nicht mehr durch empirische Entdeckungen, gleichsam wie eine Hypothese, falsifiziert werden. Er wird impliziert, oder, präziser gesagt: er bildet das grundlegende Element in einem komplexen und sich immer weiter auszudifferenzierenden Netz der sozialen, kulturellen, psychologischen,

30 Als Ausdruck einer solchen Strategie könnte man die berühmte Bemerkung Kants aus der Grundlegung zur Metaphysik der Sitten verstehen: »Ein jedes Wesen, das nicht anders als unter der Idee der Freiheit handeln kann, ist eben darum, in praktischer Hinsicht, wirklich frei (...)« (I. Kant, Grundlegung zur Metaphysik der Sitten, in: I. Kant, Die Kritiken, W. Weischedel (Hrsg.), Frankfurt am Main 1997, BA 100). Allerdings gibt sich Kant selber mit dieser »praktischen« Lösung nicht zufrieden. 
kurz gesagt: aller bewussten Verhältnissen, die sich zwischen den Menschen entfalten.

Das wahre Schlachtfeld mit dem Naturalisten ist also nicht die Debatte um psychologisch verstandene Freiheit des Willens. Freier Wille ist vielleicht die wichtigste Angelegenheit, um die es hier geht, aber das Schlachtfeld ist falsch gewählt. Man soll dem Naturalisten nicht auf der individuell-psychologischen Ebene, sondern auf dem breiten Boden des naturalistischen Dogmatismus entgegentreten. Sobald man sich stattdessen etwa mit Benjamin Libet auf naturwissenschaftlich konzipierte Experimente im Bezug auf freien Willen einlässt, ${ }^{31}$ hat man die Debatte schon längst verloren, weil man schon am An-fang viele naturalistischen Annahmen unter der Hand akzeptiert hat. Der hegelianisch gesinnte Denker will dabei nicht so sehr das von dem Naturalisten entworfene Bild, sondern dessen absoluten Anspruch auf Erklärung desavouieren. Sobald wir eine so verstandene hegelsche Perspektive annehmen, stellen sich dem Naturalisten unbequeme Fragen wegen deterministischen Annahmen, die er stillschweigend akzeptiert hat. Der Dogmatismus seiner empiristischen Methodologie, mit der er das »Problem der Willensfreiheit« zu »lösen« versucht, wird dabei offensichtlich. Wieso nimmt dieser Forscher an, dass ein solches Problem, wie Willensfreiheit, von den methodologischen Vor-aussetzungen seiner Untersuchung völlig isoliert bestimmt werden kann? Die schon im Zusammenhang mancher modernen Naturwissenschaften fragliche An-nahme einer gegenüber seinem Gegenstand absolut neutralen Untersuchungsmethodologie spitzt sich bei einem psychologisch-philosophischsozial-politisch-kulturellen Phänomen, wie das die Willensfreiheit ist, dramatisch zu.

Nehmen wir den Faden einer solchen Polemik mit dem Naturalisten auf, so treten auch die Inkonsequenzen der naturalistischen Argumentation immer mehr zutage. Es wird klar, dass empiristische Vorgehensweise im Fall der Willensfreiheit einen gewaltigen Teil unserer sozialen Erfahrung und unseres Diskurses gar nicht erklären kann. Vor allem die Tatsache, dass wir den Begriff der Gerechtigkeit anwenden und dass wir ihn für den grundlegenden Begriff der sozialen Ordnung halten. Zusammenfassend lässt sich sagen, dass eine wichtige Lehre, die wir aus Hegels perspektivistischer und holistischer Auffassung der Willensfreiheit ziehen können, die Vertiefung der theoretischen Ebene wäre, auf der die Polemik mit den naturalistischen Gegnern der Willensfreiheit aufzunehmen ist. Das Fazit lautet also: mehr an reflexiver Vorsicht, was die Voraussetzungen und Kontexte dieser Debatte betrifft. Nur so kann vermieden werden, dass durch einseitige und vereinfachende Auffassung des Phänomens

31 Siehe: B. Libet, Mind Time. Wie das Gehirn Bewusstsein produziert, Frankfurt am Main 2007. 
des freien Willens der Ausgang dieser Debatte naturalistisch vorprogrammiert wird.

JAKUB KLOC-KONKOŁOWICZ is research associate at Institute of Philosophy (Warsaw University).

Area of research:

Classical German philosophy and social philosophy.

Recent publications:

2011: »Ist der Primat des Praktischen nur »eine hohle Deklamation«? Hegelsche Kritik an Fichte in Glauben und Wissen«, in: Danz, Christian und Stolzenberg, Jürgen (Hg.): System und Systemkritik um 1800, Meiner Verlag, Hamburg, S. 239-252.

2013: »Das Ich und der Andere. Intersubjektivität in der Philosophie Johann Gottlieb Fichtes und in der Phänomenologie Edmund Husserls«, in: Stolzenberg, Jürgen - Rudolph, Oliver-Pierre (Hrsg.), Wissen, Freiheit, Geschichte. Die Philosophie Fichtes im 19. und 20. Jahrhundert. (Fichte-Studien; Band 37), Amsterdam - New York, S. 163-174.

e-mail: j.kloc-konkolowicz@uw.edu.pl 